\title{
Thermal Stability of a Reactive Hydromagnetic Poiseuille Fluid Flow through a Channel
}

\author{
Hassan Anthony Rotimi ${ }^{1, ~}$, Gbadeyan Jacob Abiodun ${ }^{2}$ \\ ${ }^{1}$ Dept. of Mathematics, Tai Solarin University of Education, Ijagun, Ogun State, Nigeria \\ ${ }^{2}$ Dept. of Mathematics, University of Ilorin, Ilorin, Nigeria
}

Email address:

anthonyhassan72@yahoo.co.uk(H. A. Rotimi),j.agbadeyan@yahoo.com(G. J. Abiodun)

\section{To cite this article:}

Hassan Anthony Rotimi, Gbadeyan Jacob Abiodun. Thermal Stability of a Reactive Hydromagnetic Poiseuille Fluid Flow through a Channel. American Journal of Applied Mathematics. Vol. 2, No. 1, 2014, pp. 14-20. doi: 10.11648/j.ajam.20140201.13

\begin{abstract}
This study is conducted to investigate the thermal stability of a reactive Hydromagnetic Poiseuille fluid flow through a channel. It is assumed that the reaction is exothermic under different chemical kinetics: Sensitized, Arrhenius and Bimolecular neglecting the concentration of the material. The analytical solutions of the nonlinear dimensionless equations governing the fluid flow are obtained using Adomian Decomposition Method (ADM) together with Pade Approximation technique to determine the thermal stability of the fluid flow and important properties of temperature fields including bifurcations and thermal criticality conditions are discussed.
\end{abstract}

Keywords: Thermal Stability, Adomian Decomposition Method (ADM), Pade Approximation, Chemical Kinetics and Hydromagnetic Fluid Flow

\section{Introduction}

In recent years, considerable attention has been paid to flow of problems of a reactive hydromagnetic fluid due to complexity of fluids in engineering and industrial applications. The fundamental works in literature have clearly shown that a number of important fluids exhibit complex behavior and there is need to understand their characteristics. For instance, [1] studied the generic criterion for the thermal stability of heat transfer fluids that was derived by drawing a control volume around arbitrary heat transfer fluid and determined the conditions which cause the fluid temperature to be stable under the condition that the fluid temperature is uniform and the fluid flow is in a steady state. Also, [2] investigated the thermal stability for a reactive viscous flow of a third - grade fluid between two parallel plates with a uniform pressure gradient (Plane - Poiseuille flow). In addition to that, [3] showed that without the axial magnetic field, and with only the torodial field due to an axial current, then the flow is strictly stable to linear axisymmetric disturbances. Moreover, [4] in their contribution also investigated the inherent irreversibility and thermal stability in the flow of a variable viscosity fluid through a cylindrical pipe with convective cooling. Recently, in the work of [5], he focused attention on the influence of magnetic field on the flow thermal structure where the effect of magnetic field on the development of thermal instability was considered.

Mathematically speaking, the thermal boundary layer equation for a reactive hydromagnetic fluid flow constitutes a nonlinear problem and long - term behaviour of the solutions which will provide us an insight into the complex physical process of thermal instability in the system as described in [1]. The theory of nonlinear differential equations is quite elaborate and their solution remains an extremely important problem of practical relevance in sciences and engineering.

Moreover, the determination of thermal criticality in a reactive hydromagnetic flow system especially with the involvement of Joule heating is extremely important from the application point of view. Thermal criticality occurs when the rate of heat generation within the system exceed heat dissipation to the surroundings as mentioned in [6]. This condition is incipient thermal runaway or ignition in the flow system as discussed in [7] and the primary aim of thermal criticality analysis is the prediction of the critical or unsafe flow conditions in order to avoid them.

Keeping this in view, the present study investigates the thermal stability of a reactive hydromagnetic Poiseuille fluid flow through a channel of different chemical kinetics namely: Sensitized, Arrhenius and Bimolecular kinetics and to show that there exist multiple solutions. To achieve this, $\mathrm{ADM}$, together with Pade Approximation will be used to 
obtain the solution of the nonlinear differential equations governing the fluid flow.

In the rest of this paper, the problem is formulated in section 2 and the governing equations are solved in section 3 using ADM and Pade Approximation. Presentation of analytical results of the problems is shown in tables, and graphs in section 4 and section 5 gives the concluding remarks.

\section{Mathematical Model}

We consider the steady flow of a reactive, incompressible and electrically conducting fluid flow through a channel between two parallel plates with isothermal wall temperature under the influence of a transverse magnetic field strength $B_{0}$. Neglecting the consumption of the reactant, the dimensionless governing equations of the momentum and energy equations from [6] are:

$$
\begin{gathered}
\frac{d^{2} u}{d y^{2}}-H^{2} u+G=0 \\
\left.\frac{d^{2} T}{d y^{2}}+\lambda\left[\begin{array}{l}
(1+\delta T)^{m} e^{\frac{T}{1+\delta T}} \\
+\gamma\left(\left(\frac{d u}{d y}\right)^{2}+H^{2} u^{2}\right.
\end{array}\right)\right]=0
\end{gathered}
$$

Together with the following boundary conditions

$$
\begin{aligned}
\frac{d u}{d y}=\frac{d T}{d y}=0 \quad \text { on } y & =0 \quad \text { and } \\
u & =T=0 \text { on } y=1
\end{aligned}
$$

The following dimensionless parameters have been used for the above equations (1) - (3)

$$
\begin{aligned}
& y=\frac{\bar{y}}{a}, \quad u=\frac{\bar{u}}{U}, \quad T=\frac{E\left(\bar{T}-T_{0}\right)}{R T_{0}^{2}}, \quad P=\frac{a \bar{P}}{\mu U}, \\
& \lambda=\frac{Q E A a^{2} C_{0}}{k R T_{0}^{2}}\left(\frac{k T_{0}}{v l}\right)^{m} e^{-\frac{E}{R T_{0}}}, \quad \delta=\frac{R T_{0}}{E}, \quad H^{2}=\frac{\sigma B_{0}^{2} a^{2}}{\mu}, \\
& G=-\frac{d P}{d x}, B r=\frac{E \mu U^{2}}{k R T_{0}^{2}}, \text { and }
\end{aligned}
$$

$$
\gamma=\frac{\mu U^{2} e^{\frac{E}{R T_{0}}}}{Q A a^{2} C_{0}}
$$

where the additional chemical kinetics term in the energy equation is due to [7]. Here $u$ is axial velocity, $T$ represents the fluid temperature, $a$ is channel half width, $C_{o}$ is reactant species initial concentration, $E$ is activation energy, $R$ is the universal gas constant, $A$ is reaction rate constant, $k$ thermal conductivity coefficient, $\mu$ is fluid viscosity, $Q$ is the heat of reaction term, $P$ is the modified pressure, $l$ is the Planck's number, $v$ is the vibration frequency, $\sigma_{0}$ represents electrical conductivity. Also $G$ represents constant axial pressure gradient, $H$ represents Hartmann number, $\lambda$ is Frank - Kamenettski parameter and $\delta$ is the activation energy parameter, $\gamma$ is viscous heating parameter and $m$ is a numerical constant such that $m \in\{-2,0,0.5\}$. The three values taken by the parameter $m$ represent the numerical exponent for Sensitized, Arrhenius and Bimolecular kinetics respectively.

\section{Method of Solutions}

\subsection{Adomian Decomposition Method (ADM)}

The use of ADM has been applied to a wide class of problems in sciences. Literature for ADM can be found in [8], [9], [10], [11] and [12]. The method has shown reliable results in supplying analytical approximations that converge very rapidly as reported in [13].

Solving (1) with the appropriate boundary conditions, one obtains,

$$
u(y)=\frac{G}{H^{2}}\left(1-\frac{\operatorname{Cosh}(H y)}{\operatorname{Cosh}(H)}\right)
$$

\subsubsection{Case 1: When $m=-2$ (Sensitized Kinetics)}

Substituting (5) and making $m=-2$ in equation (2), we have:

$$
\frac{d^{2} T}{d y^{2}}+\lambda\left[\begin{array}{l}
\frac{e^{\frac{T}{1+\delta T}}}{(1+\delta T)^{2}} \\
+\frac{\gamma G^{2}}{H^{2}}\left(\begin{array}{l}
\frac{\operatorname{Cosh}(2 H y)}{\operatorname{Cosh}{ }^{2} H}- \\
\frac{2 \operatorname{Cosh}(H y)}{\operatorname{Cosh}(H)}+1
\end{array}\right)
\end{array}\right]=0
$$

Integrating (6) twice, we have

$$
T(y)=a_{0}-\lambda \int_{0}^{y} \int_{0}^{y}\left[\begin{array}{l}
\frac{e^{\frac{T}{1+\delta T}}}{(1+\delta T)^{2}} \\
+\frac{\gamma G^{2}}{H^{2}}\left(\begin{array}{l}
\frac{\operatorname{Cosh}(2 H y)}{\operatorname{Cosh}{ }^{2} H} \\
-\frac{2 \operatorname{Cosh}(H y)}{\operatorname{Cosh}(H)} \\
+1
\end{array}\right)
\end{array}\right] d y d y
$$

where $a_{0}=T(0)$ to be determined by using the other boundary conditions. Introducing the series solutions of $\mathrm{ADM}$ in the form

$$
T(y)=\sum_{n=0}^{\infty} T_{n}(y)
$$

such that (8) in (7) gives

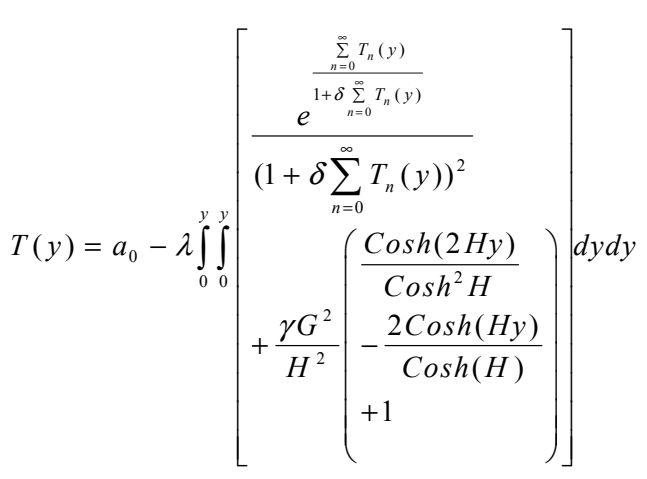


We let the nonlinear term be represented by

$$
\sum_{n=0}^{\infty} A_{n}(y)=\frac{e^{\frac{\sum_{n=0}^{\infty} T_{n}(y)}{1+\delta \sum_{n=0}^{\infty} T_{n}(y)}}}{\left(1+\delta \sum_{n=0}^{\infty} T_{n}(y)\right)^{2}}
$$

such that

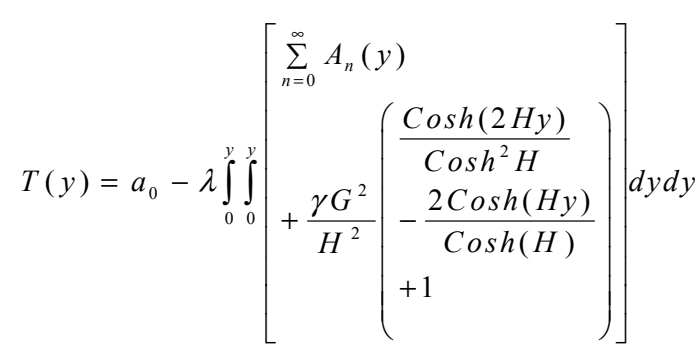

Obtaining few terms of Adomian polynomials as

$$
\begin{aligned}
& \mathrm{A}_{0}=\frac{e^{\frac{T_{0}(y)}{1+\delta T_{0}(y)}}}{\left[1+\delta T_{0}(y)\right]^{2}}, \\
& \mathrm{~A}_{1}=\frac{e^{\frac{T_{0}(y)}{1+\delta T_{0}(y)}}\left(1-2 \delta-2 \delta^{2} T_{0}(y)\right) T_{1}}{\left[1+\delta T_{0}(y)\right]^{4}} \\
& \mathrm{~A}_{2}=\frac{e^{\frac{T_{0}(y)}{1+\delta T_{0}(y)}}}{2\left[1+\delta T_{0}(y)\right]^{6}}\left[\begin{array}{l}
\left(\begin{array}{l}
1-6 \delta+6 \delta^{2} \\
+6 \delta^{2}(2 \delta-1) T_{0}(y) \\
+6 \delta^{4} T_{0}(y)^{2} \\
-2\left(1+\delta T_{0}(y)^{2}\left(-1+2 \delta+2 \delta^{2}\right) T_{0}(y) T_{2}(y)\right)
\end{array}\right) T_{1}(y)^{2}
\end{array}\right)
\end{aligned}
$$

Taking the zeroth component of (11) following the new modification following [8] and [10]:

$$
\begin{aligned}
& T_{0}(y)=a_{0} \\
& T_{1}(y)=-\lambda \int_{0}^{y} \int_{0}^{y}\left[A_{0}(y)+\frac{\gamma G^{2}}{H^{2}}\left(\begin{array}{c}
\frac{\operatorname{Cosh}(2 H y)}{\operatorname{Cosh}{ }^{2} H} \\
-\frac{2 \operatorname{Cosh}(H y)}{\operatorname{Cosh}(H)}+1
\end{array}\right)\right] d y d y \\
& T_{n+1}(y)=\lambda \int_{0}^{y} \int_{0}^{y}\left[A_{n}(y)\right] d y d y, n \geq 1
\end{aligned}
$$

The approximate solutions are given by the partial sum when $k=2$

$$
T(y)=\sum_{n=0}^{k} T_{n}(y)
$$

such that

$$
T_{0}(y)=a_{0}
$$

$$
\begin{aligned}
& T_{1}(y)=-\lambda\left(\begin{array}{l}
\frac{G^{2} \gamma}{2 H^{2}}\left[\begin{array}{l}
y^{2} \\
+\frac{1}{H^{2}}\left(\begin{array}{l}
\operatorname{Sech}[H](4 \\
-4 \operatorname{Cosh}[H y] \\
\left.+\operatorname{Sech}[H] \operatorname{Sinh}[H y]^{2}\right)
\end{array}\right)
\end{array}\right) \\
+\frac{\mathrm{e}^{\frac{a_{0}}{1+\delta a_{0}} y^{2}}}{2\left(1+\delta a_{0}\right)^{2}}
\end{array}\right) \\
& T_{2}(y)=-\frac{\mathrm{e}^{\frac{a_{0}}{1+\delta_{a_{0}}}} \gamma G^{2} \lambda^{2}\left[2 \delta^{2} a_{0}+2 \delta-1\right]}{24 H^{2}\left(1+\delta a_{0}\right)^{4}}\left(y^{4}+\frac{1}{H a^{4}}\left[\begin{array}{l}
3 \operatorname{Sech}[H](16-16 \operatorname{Cosh}[H y] \\
-H^{2} y^{2}(-8+\operatorname{Sech}[H]) \\
\left.+\operatorname{Sech}[H] \operatorname{Sinh}[H]^{2}\right)
\end{array}\right]\right) \\
& -\frac{\mathrm{e}^{\frac{2 a_{0}}{1+\delta_{a_{0}}}} \gamma G^{2} \lambda^{2}\left[2 \delta^{2} a_{0}+2 \delta-1\right]}{24 H^{2}\left(1+\delta a_{0}\right)^{6}} y^{4}
\end{aligned}
$$

and the solution for the temperature under sensitized kinetics $T_{s}(y)$ is thus given as:

$$
\begin{aligned}
& T_{S}(y)=a_{0}-\lambda\left(\begin{array}{l}
\frac{G^{2} \gamma}{2 H^{2}}\left[\begin{array}{l}
y^{2} \\
+\frac{1}{H^{2}}\left(\begin{array}{l}
\operatorname{Sech}[H](4 \\
-4 \operatorname{Cosh}[H y] \\
\left.+\operatorname{Sech}[H] \operatorname{Sinh}[H y]^{2}\right)
\end{array}\right)
\end{array}\right) \\
+\frac{\mathrm{e}^{\frac{a_{0}}{1+\delta a_{0}}} y^{2}}{2\left(1+\delta a_{0}\right)^{2}}
\end{array}\right) \\
& -\frac{\mathrm{e}^{\frac{a_{0}}{1+\delta \delta_{0}}} \gamma G^{2} \lambda^{2}\left[2 \delta^{2} a_{0}+2 \delta-1\right]}{24 H^{2}\left(1+\delta a_{0}\right)^{4}}\left(y^{4}+\frac{1}{H^{4}}\left[\begin{array}{l}
3 \operatorname{Sech}[H](16-16 \operatorname{Cosh}[H y] \\
-H^{2} y^{2}(-8+\operatorname{Sech}[H]) \\
\left.+\operatorname{Sech}[H] \operatorname{Sinh}[H y]^{2}\right)
\end{array}\right]\right) \\
& -\frac{\mathrm{e}^{\frac{2 a_{0}}{1+\delta a_{0}}} \gamma G^{2} \lambda^{2}\left[2 \delta^{2} a_{0}+2 \delta-1\right]}{24 H^{2}\left(1+\delta a_{0}\right)^{6}} y^{4}
\end{aligned}
$$

\subsubsection{Case 2: When $m=0$ (Arrhenius Kinetics)}

In a similar way, substituting (5) and making $m=0$ in equation (2), we have

$$
\frac{d^{2} T}{d y^{2}}+\lambda\left[e^{\frac{T}{1+\delta T}}+\frac{\gamma G^{2}}{H^{2}}\left(\begin{array}{l}
\frac{\operatorname{Cosh}(2 H y)}{\operatorname{Cosh} H} \\
-\frac{2 \operatorname{Cosh}(H y)}{\operatorname{Cosh}(H)}+1
\end{array}\right)\right]=0
$$

In a similar way, we integrate (16) twice to obtain

$$
T(y)=b_{0}-\lambda \int_{0}^{y} \int_{0}^{y}\left[e^{\frac{T}{1+\delta T}}+\frac{\gamma G^{2}}{H^{2}}\left(\begin{array}{l}
\frac{\operatorname{Cosh}(2 H y)}{\operatorname{Cosh}{ }^{2} H} \\
-\frac{2 \operatorname{Cosh}(H y)}{\operatorname{Cosh}(H)}+1
\end{array}\right)\right] d y d y
$$

where $b_{0}=T(0)$ to be determined by using the other boundary conditions. Introducing the same series solutions (8) such that (17) becomes

$$
T(y)=b_{0}-\lambda \int_{0}^{y} \int_{0}^{y}\left[e^{\frac{\sum_{n=0}^{\infty} T_{n}(y)}{1+\delta \sum_{n=0}^{\infty} T_{n}(y)}}+\frac{\gamma G^{2}}{H^{2}}\left(\begin{array}{l}
\frac{\operatorname{Cosh}(2 H y)}{\operatorname{Cosh}{ }^{2} H} \\
-\frac{2 \operatorname{Cosh}(H y)}{\operatorname{Cosh}(H)}+1
\end{array}\right)\right] d y d y(
$$

Here we let the nonlinear term be represented by 


$$
\sum_{n=0}^{\infty} B_{n}(y)=e^{\frac{\sum_{n=0}^{\infty} T_{n}(y)}{1+\delta \sum_{n=0}^{\infty} T_{n}(y)}}
$$

such that

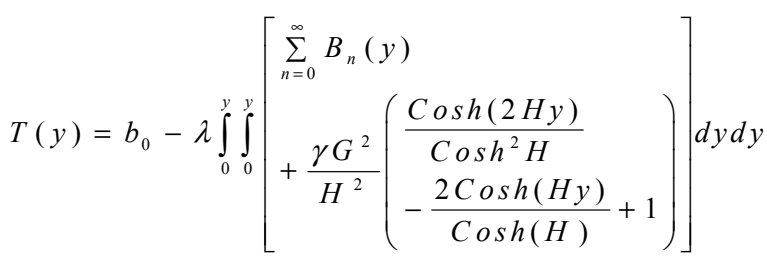

Obtaining few terms of Adomian polynomials as

$\mathrm{B}_{0}=e^{\frac{T_{0}(y)}{1+\delta T_{0}(y)}}$

$\mathrm{B}_{1}=\frac{e^{\frac{T_{0}(y)}{1+\delta T_{0}(y)}} T_{1}(y)}{\left[1+\delta T_{0}(y)\right]^{2}}$

$$
\mathrm{B}_{2}=\frac{\left.e^{\frac{T_{0}(y)}{1+\delta \delta_{0}(y)}}\left[\left(1-2 \delta-2 \delta^{2} T_{0}(y)\right)\right) T_{1}(y)^{2}+2\left(1+\delta T_{0}(y)^{2} T_{2}(y)\right)\right]}{2\left[1+\delta T_{0}(y)\right]^{4}}
$$

Taking the zeroth component of (20) following the new modification from literature as follows:

$$
\begin{aligned}
& T_{0}(y)=b_{0} \\
& T_{1}(y)=-\lambda \int_{0}^{y} \int_{0}^{y}\left[B_{0}(y)+\frac{\gamma G^{2}}{H^{2}}\left(\frac{\operatorname{Cosh}(2 H y)}{\operatorname{Cosh}^{2} H}-\frac{2 \operatorname{Cosh}(H y)}{\operatorname{Cosh}(H)}+1\right)\right] d y d y \\
& T_{n+1}(y)=\lambda \int_{0}^{y} \int_{0}^{y}\left[B_{n}(y)\right] d y d y, n \geq 1
\end{aligned}
$$

such that

$$
\begin{aligned}
& T_{0}(y)=b_{0} \\
& T_{1}(y)=-\lambda\left(\frac{1}{2} \mathrm{e}^{\frac{b_{0}}{1+\delta_{0}}} y^{2}+\frac{\gamma G^{2}}{2 H^{2}}\left[y^{2}+\frac{\gamma G^{2}}{H^{2}}\left(\begin{array}{l}
\operatorname{Sech}[H](4-4 \operatorname{Cosh}[H y] \\
\left.+\operatorname{Sech}[H] \operatorname{Sinh}[H y]^{2}\right)
\end{array}\right)\right]\right) \\
& \left.T_{2}(y)=\frac{\mathrm{e}^{\frac{b_{0}}{1+\delta \delta_{0}}} \lambda^{2}}{24\left(1+\delta b_{0}\right)^{2}}\left[\begin{array}{l}
\mathrm{e}^{\frac{b_{0}}{1+\delta \delta_{0}}} y^{4}+ \\
\frac{G^{2} \gamma}{H^{2}}\left(y^{4}+\frac{1}{H^{4}}\left(\begin{array}{l}
3 \operatorname{Sech}[H](16-16 \operatorname{Cosh}[H y] \\
-H a^{2} y^{2}(-8+\operatorname{Sech}[H]) \\
\left.+\operatorname{Sech}[H] \operatorname{Sinh}[H y]^{2}\right)
\end{array}\right)\right.
\end{array}\right)\right]
\end{aligned}
$$

The approximate solution is given by the partial sum as in (14a) such that the solution for the temperature under Arrhenius kinetics $T_{A}(y)$ is thus given as:

$$
\begin{aligned}
& T_{A}(y)=b_{0}-\lambda\left[\begin{array}{l}
\frac{\gamma G^{2}}{2 H^{4}}\left(y^{2}+\operatorname{Sech}(H)\left(4-4 \operatorname{Cosh}(H y)+\operatorname{Sech}(H) \operatorname{Sinh}^{2}(H y)\right)\right. \\
+\frac{e^{\frac{b_{0}}{1+\delta b_{0}}} y^{2}}{2\left(1+\delta b_{0}\right)^{2}}
\end{array}\right] \\
& +\frac{\mathrm{e}^{\frac{b_{0}}{1+\delta b_{0}}} \lambda^{2}}{24\left(1+\delta b_{0}\right)^{2}}\left[\mathrm{e}^{\frac{b_{0}}{1+\delta b_{0}}} y^{4}+\frac{\gamma G^{2}}{H^{2}}\left(y^{4}+\frac{1}{H^{4}}\left(\begin{array}{l}
3 \operatorname{Sech}[H](16-16 \operatorname{Cosh}[H y] \\
-H^{2} y^{2}(-8+\operatorname{Sech}[H]) \\
\left.+\operatorname{Sech}[H] \operatorname{Sinh}[H y]^{2}\right)
\end{array}\right)\right)\right]
\end{aligned}
$$

3.1.3 Case 3: When $\mathrm{m}=0.5$ (Bimolecular Kinetics)

In a similar way, substituting (5) and making $m=0.5$ in equation (2), we have

$$
\frac{d^{2} T}{d y^{2}}+\lambda\left[\begin{array}{l}
(\sqrt{(1+\delta T)}) e^{\frac{T}{1+\delta T}} \\
+\frac{\gamma G^{2}}{H^{2}}\left(\frac{\operatorname{Cosh}(2 H y)}{\operatorname{Cosh}^{2} H}-\frac{2 \operatorname{Cosh}(H y)}{\operatorname{Cosh}(H)}+1\right)
\end{array}\right]=0
$$

Integrating (24) twice, in a similar way as done before, we have:

$$
T(y)=d_{0}-\lambda \int_{0}^{y} \int_{0}^{y}\left[\begin{array}{l}
(\sqrt{(1+\delta T)}) e^{\frac{T}{1+\delta T}} \\
+\frac{\gamma G^{2}}{H^{2}}\left(\begin{array}{l}
\frac{\operatorname{Cosh}(2 H y)}{\operatorname{Cosh} \operatorname{Sosh}^{2}} \\
-\frac{2 \operatorname{Cosh}(H y)}{\operatorname{Cosh}(H)}+1
\end{array}\right)
\end{array}\right) d y d y
$$

where $d_{0}=T(0)$ to be determined by using the other boundary conditions. Introducing the series solutions (8) in a similar way such that (25) becomes

$$
T(y)=d_{0}-\lambda \int_{0}^{y} \int_{0}^{y}\left[\begin{array}{l}
\left(\sqrt{\left(1+\delta \sum_{n=0}^{\infty} T_{n}(y)\right)}\right) e^{\frac{\sum_{n=0}^{\infty} T_{n}(y)}{1+\delta \sum_{n=0}^{\infty} T_{n}(y)}} \\
+\frac{\gamma G^{2}}{H^{2}}\left(\begin{array}{c}
\frac{\operatorname{Cosh}(2 H y)}{\operatorname{Cosh} H}- \\
\frac{2 \operatorname{Cosh}(H y)}{\operatorname{Cosh}(H)}+1
\end{array}\right)
\end{array}\right] d y d y
$$

We let the nonlinear term be represented by

$$
\sum_{n=0}^{\infty} D_{n}(y)=\left(\sqrt{\left(1+\delta \sum_{n=0}^{\infty} T_{n}(y)\right)} e^{\frac{\sum_{n=0}^{\infty} T_{n}(y)}{1+\delta \sum_{n=0}^{\infty} T_{n}(y)}}\right.
$$

such that

$$
T(y)=d_{0}-\lambda \int_{0}^{y} \int_{0}^{y}\left[\sum_{n=0}^{\infty} D_{n}(y)+\frac{\gamma G^{2}}{H^{2}}\left(\begin{array}{l}
\frac{\operatorname{Cosh}(2 H y)}{\operatorname{Cosh}{ }^{2} H} \\
-\frac{2 \operatorname{Cosh}(H y)}{\operatorname{Cosh}(H)}+1
\end{array}\right)\right] d y d y
$$

Obtaining few terms of Adomian polynomials as

$$
\begin{aligned}
& \mathrm{D}_{0}=e^{\frac{T_{0}(y)}{1+\delta T_{0}(y)}} \sqrt{\left[1+\delta T_{0}(y)\right]}, \\
& \mathrm{D}_{1}=\frac{e^{\frac{T_{0}(y)}{1+\delta T_{0}(y)}}\left(2+\delta+\delta^{2} T_{0}(y)\right) T_{1}(y)}{2\left[1+\delta T_{0}(y)\right]^{\frac{3}{2}}}, \\
& \mathrm{D}_{2}=\frac{e^{\frac{T_{0}(y)}{1+\delta T_{0}(y)}}}{8\left[1+\delta T_{0}(y)\right]^{\frac{7}{2}}}\left[\begin{array}{l}
\left(-\left(-4+4 \delta+\delta^{2}+2 \delta^{2}(2+\delta) T_{0}(y)\right.\right. \\
\left.+\delta^{4} T_{0}(y)^{2}\right) T_{1}(y)^{2} \\
+4\left(1+\delta T_{0}(y)\right)^{2}\left(2+\delta+\delta^{2} T_{0}(y)\right) T_{2}(y)
\end{array}\right]
\end{aligned}
$$

Taking the zeroth component of (28) following the new modification from literature as follows:

$T_{0}(y)=d_{0}$ 


$$
\begin{gathered}
T_{1}(y)=-\lambda \int_{0}^{y} \int_{0}^{y}\left[D_{0}(y)+\frac{\gamma G^{2}}{H^{2}}\left(\frac{\operatorname{Cosh}(2 H y)}{\operatorname{Cosh}{ }^{2} H}-\frac{2 \operatorname{Cosh}(H y)}{\operatorname{Cosh}(H)}+1\right)\right] d y d y \\
T_{n+1}(y)=\lambda \int_{0}^{y} \int_{0}^{y}\left[D_{n}(y)\right] d y d y, n \geq 1
\end{gathered}
$$

such that

$$
\begin{aligned}
& T_{0}(y)=d_{0} \\
& T_{1}(y)=\frac{-\lambda \gamma G^{2}}{2 H a^{2}}\left(y^{2}+\frac{1}{H^{2}}\left[\begin{array}{l}
\operatorname{Sech}[H a](4-4 \operatorname{Cosh}[H a y] \\
+\operatorname{Sech}[H a] \operatorname{Sinh}[H a y]^{2}
\end{array}\right]\right. \\
& -\frac{\lambda}{2} \mathrm{e}^{\frac{d_{0}}{1+\delta d_{0}}} y^{2} \sqrt{1+\delta d_{0}}
\end{aligned}
$$

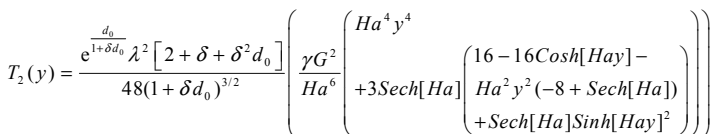

$$
\begin{aligned}
& +\frac{\lambda^{2}\left[2+\delta+\delta^{2} d_{0}\right]}{48\left(1+\delta d_{0}\right)^{3 / 2}} \mathrm{e}^{\frac{2 d_{0}}{1+\delta d_{0}}} y^{4} \sqrt{1+\delta d_{0}}
\end{aligned}
$$

Hence, the approximate solution is given by the partial sum as in (14a) such that the solution for the temperature under Bimolecular kinetics $T_{B}(y)$ is thus given as:

$$
\begin{aligned}
& T_{B}(y)=d_{0}-\frac{\lambda \gamma G^{2}}{2 H^{2}}\left(y^{2}+\frac{\operatorname{Sech}[H]\left(4-4 \operatorname{Cosh}[H y]+\operatorname{Sech}[H] \operatorname{Sinh}[H y]^{2}\right)}{H^{2}}\right) \\
& +\frac{\mathrm{e}^{\frac{d_{0}}{\mathrm{e}^{+\delta_{0}}}} \lambda^{2}\left[2+\delta+\delta^{2} d_{0}\right]}{48\left(1+\delta d_{0}\right)^{3 / 2}}\left(\frac{\gamma G^{2}}{H^{6}}\left(H^{4} y^{4}+3 \operatorname{Sech}[H]\left(\begin{array}{l}
16-16 \operatorname{Cosh}[H y] \\
-H^{2} y^{2}(-8+\operatorname{Sech}[H]) \\
+\operatorname{Sech}[H] \operatorname{Sinh}[H y]^{2}
\end{array}\right)\right)\right) \\
& +\frac{\lambda^{2}\left[2+\delta+\delta^{2} d_{0}\right]}{48\left(1+\delta d_{0}\right)^{3 / 2}} \mathrm{e}^{\frac{2 d_{0}}{1+\delta d_{0}}} y^{4} \sqrt{1+\delta d_{0}}-\frac{\lambda}{2} \mathrm{e}^{\frac{d_{0}}{1+\delta d_{0}}} y^{2} \sqrt{1+\delta d_{0}}
\end{aligned}
$$

\subsection{Pade Approximation}

The Padé approximation is a rational function that can be thought of as a generalization of a Taylor polynomial. The continuation of solutions using Pade approximants has been shown in [5] to be more reliable and convergent on the entire real axis if the underlying series solution is free of singularities on the real axis. Using the built - in Pade Approximant procedure in MATHEMATICA, the diagonal form of the series solutions (15), (23) and (31) are evaluated respectively at $y=1$ so as to get expressions for the unknown constants $a_{0}, b_{0}$ and $d_{0}$ using the boundary conditions

$$
T(1)=0
$$

Taking the diagonal Pade approximants $[\mathrm{M} / \mathrm{M}]$ of (32) at various values of $\mathrm{M}$ leads to an eigenvalue problem. To show that the series converge, the unknown constants are evaluated using values for the known parameters of different chemical kinetics as shown in tables $1-3$. The critical values of Frank - Kameneskii parameter $\left(\lambda_{c}\right)$ for non - existence of solution or thermal runaway for each chemical kinetics are also presented in Tables $4-6$.

\section{Results and Discussion}

Table 1: Numerical values for $a_{0}$

\begin{tabular}{ccccc}
\hline Pade & $\mathbf{H}$ & $\boldsymbol{\lambda}$ & $\boldsymbol{T}_{\text {Lower }}$ & $\boldsymbol{T}_{\text {Upper }}$ \\
\hline $2 / 2$ & 1 & 0.5 & -0.3190 & 0.2466 \\
$5 / 5$ & 1 & 0.5 & -0.7018 & 0.2464 \\
$10 / 10$ & 1 & 0.5 & -1.8924 & 0.2464 \\
$15 / 15$ & 1 & 0.5 & -1.8750 & 0.2464 \\
$20 / 20$ & 1 & 0.5 & -1.8745 & 0.2464 \\
$25 / 25$ & 1 & 0.5 & -1.8748 & 0.2464 \\
$30 / 30$ & 1 & 0.5 & -1.8748 & 0.2464 \\
$50 / 50$ & 1 & 0.5 & -1.8748 & 0.2464 \\
\hline
\end{tabular}

Tables $1-3$ present the rapid convergence of the two solutions (upper and lower) for different chemical kinetics. Tables $4-6$ also show the effects of magnetic intensity and viscous heating on the development of thermal runaway for different chemical kinetics. An increase in Hartmann $(H)$ number gives an increase in the critical value of Frank Kameneskii parameter $\left(\lambda_{c}\right)$ which stabilizes the flow while an increase in viscous heating parameter $(\gamma)$ gives a decrease in the critical value $\left(\lambda_{c}\right)$

Table 2: Numerical values for $b_{0}$

\begin{tabular}{ccccc}
\hline Pade & $\mathbf{H}$ & $\boldsymbol{\lambda}$ & $\boldsymbol{T}_{\text {Lower }}$ & $\boldsymbol{T}_{\text {Upper }}$ \\
\hline $2 / 2$ & 1 & 0.5 & 0.3581 & 3.8254 \\
$5 / 5$ & 1 & 0.5 & 0.3578 & 0.6132 \\
$10 / 10$ & 1 & 0.5 & -1.6206 & 0.3578 \\
$15 / 15$ & 1 & 0.5 & -1.6207 & 0.3578 \\
$20 / 20$ & 1 & 0.5 & -1.6206 & 0.3578 \\
$25 / 25$ & 1 & 0.5 & -1.6206 & 0.3578 \\
$30 / 30$ & 1 & 0.5 & -1.6206 & 0.3578 \\
$50 / 50$ & 1 & 0.5 & -1.6206 & 0.3578 \\
\hline
\end{tabular}

Table 3: Numerical values for $d_{0}$

\begin{tabular}{ccccc}
\hline Pade & $\mathbf{H}$ & $\lambda$ & $\boldsymbol{T}_{\text {Lower }}$ & $\boldsymbol{T}_{U_{\text {pper }}}$ \\
\hline $2 / 2$ & 1 & 0.5 & 0.4181 & 0.7387 \\
$5 / 5$ & 1 & 0.5 & -0.9003 & 0.4183 \\
$10 / 10$ & 1 & 0.5 & -2.3467 & 0.4183 \\
$15 / 15$ & 1 & 0.5 & -1.1293 & 0.4183 \\
$20 / 20$ & 1 & 0.5 & -1.7360 & 0.4183 \\
$60 / 60$ & 1 & 0.5 & -1.4864 & 0.4183 \\
$80 / 80$ & 1 & 0.5 & -1.0624 & 0.4183 \\
$120 / 120$ & 1 & 0.5 & -1.0624 & 0.4183 \\
\hline
\end{tabular}


Table 4: Effect of different parameters on the development of thermal runaway for Sensitized kinetics

\begin{tabular}{cccccc}
\hline Pade & $\mathbf{H}$ & $\boldsymbol{\gamma}$ & $\boldsymbol{\delta}$ & $\mathbf{G}$ & $\boldsymbol{\lambda}_{\mathbf{c}}$ \\
\hline $2 / 2$ & 1 & 1 & 1 & 1 & -0.2037 \\
$2 / 2$ & 2 & 1 & 1 & 1 & 0.1610 \\
$2 / 2$ & 3 & 1 & 1 & 1 & 0.3220 \\
$2 / 2$ & 1 & 1 & 1 & 1 & -0.2037 \\
$2 / 2$ & 1 & 2 & 1 & 1 & 0.1537 \\
$2 / 2$ & 1 & 3 & 1 & 1 & 0.3075 \\
\hline
\end{tabular}

Table 5: Effect of different parameters on the development of thermal runaway for Arrhenius kinetics

\begin{tabular}{cccccc}
\hline Pade & $\mathbf{H}$ & $\boldsymbol{\gamma}$ & $\boldsymbol{\delta}$ & $\mathbf{G}$ & $\boldsymbol{\lambda}_{\mathbf{c}}$ \\
\hline $2 / 2$ & 1 & 1 & 1 & 1 & 1.296 \\
$2 / 2$ & 2 & 1 & 1 & 1 & 1.338 \\
$2 / 2$ & 3 & 1 & 1 & 1 & 1.384 \\
$2 / 2$ & 1 & 1 & 1 & 1 & 1.296 \\
$2 / 2$ & 1 & 2 & 1 & 1 & 1.162 \\
$2 / 2$ & 1 & 3 & 1 & 1 & 1.048 \\
\hline
\end{tabular}

Table 6: Effect of different parameters on the development of thermal runaway for bimolecular kinetics

\begin{tabular}{cccccc}
\hline Pade & $\mathbf{H}$ & $\boldsymbol{\gamma}$ & $\boldsymbol{\delta}$ & $\mathbf{G}$ & $\boldsymbol{\lambda}_{\mathbf{c}}$ \\
\hline $2 / 2$ & 1 & 1 & 1 & 1 & 1.617 \\
$2 / 2$ & 2 & 1 & 1 & 1 & 1.622 \\
$2 / 2$ & 3 & 1 & 1 & 1 & 1.623 \\
$2 / 2$ & 1 & 1 & 1 & 1 & 1.617 \\
$2 / 2$ & 1 & 2 & 1 & 1 & 1.609 \\
$2 / 2$ & 1 & 3 & 1 & 1 & 1.596 \\
\hline
\end{tabular}

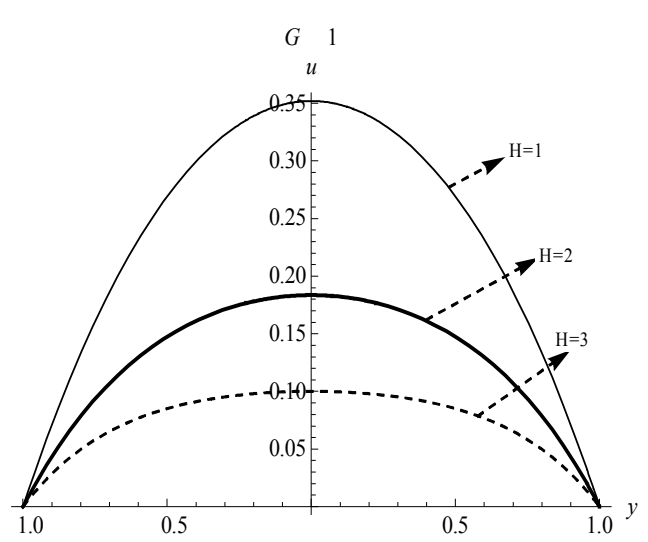

Figure 1: Velocity profile with variation in Hartmann number

Figure 1 shows the plot of velocity profile for variation in Hartmann number $(H)$. As observed, maximum velocity occurs at the minimum value of parameter, that is, further increase in $H$ decreases the flow velocity maximum; this is due to the presence of Lorentz forces which has retarding effects on fluid flow when placed across the flow channel. Figure 2 depicts the temperature profile for different chemical kinetics, from the plot it is clearly observed that the maximum temperature is obtained as the numerical exponent $(m)$ increases from -2 to 0.5 . It is generally noticed that the fluid temperature is zero at both upper and lower stationary surfaces of the channel while the maximum temperature occurs at the centreline of the channel.

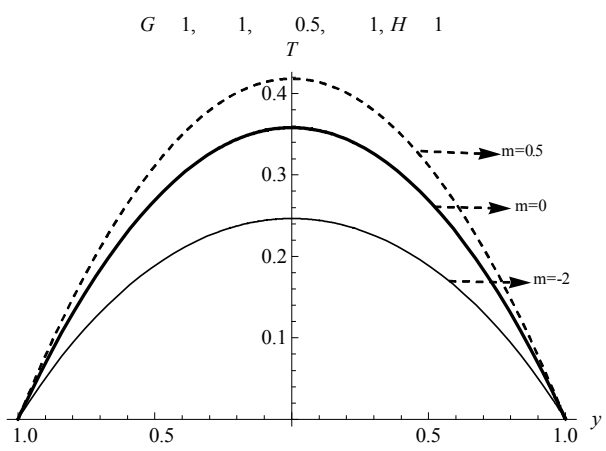

Figure 2: Temperature profile for different chemical kinetics

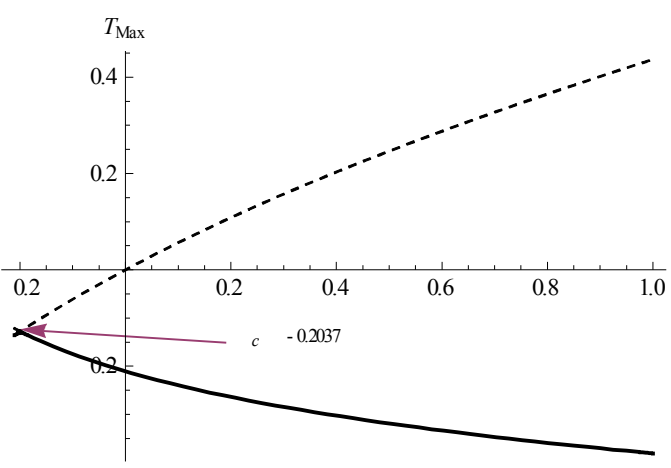

Figure 3: Solution branches for Sensitized kinetics

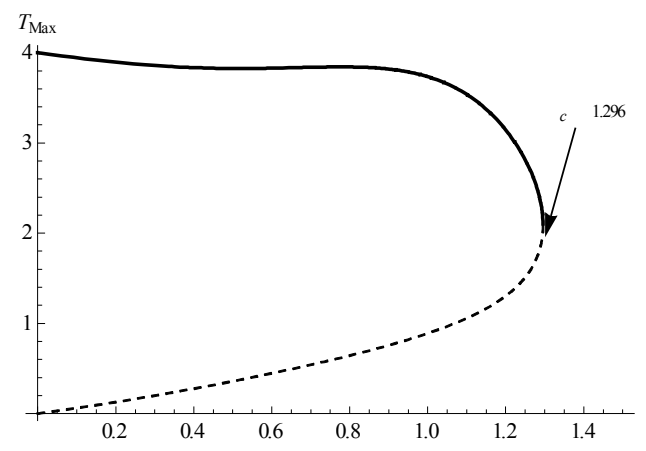

Figure 4: Solution branches for Arrhenius kinetics

Figures 3 -5 show each solution branches diagram for different chemical kinetics. These plots represent the qualitative change in the flow system as Frank - Kameneskii parameter $(\lambda)$ increases with respective numerical exponents $(m)$ increases from -2 to 0.5 . In particular, $0 \leq \lambda \leq 1$ there is a critical value, $\lambda_{c}$ (turning point), such that for $0<\lambda<\lambda_{c}$, there are two solutions. The upper 
and lower branches occur due to the non linear nature of the governing thermal boundary layer equation (2). In the plots, for $\lambda_{c}<\lambda$, the system has no real solution and display classical forms indicating thermal runaway.

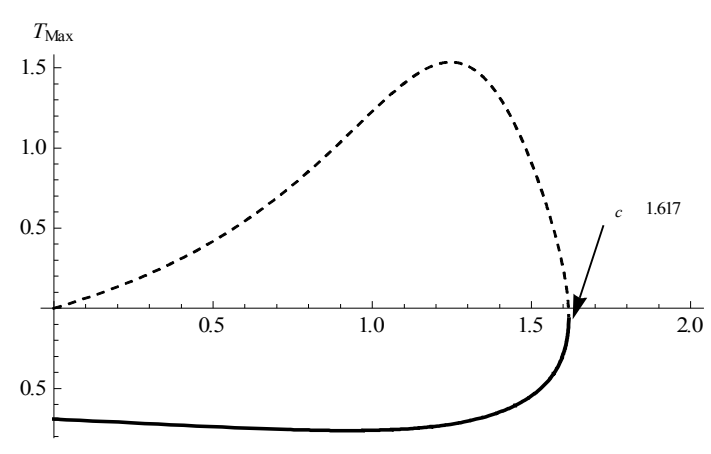

Figure 5: Solution branches for bimolecular kinetics

\section{Conclusion}

In this paper, the nonlinear differential equations governing the fluid flow are solved using ADM, and Pade Approximant is employed to investigate the thermal stability of a reactive hydromagnetic fluid flow through a channel for different chemical kinetics. The solution reveals accurately the thermal stability conditions and various solution branches. It is observed that, the maximum temperature is obtained as the numerical exponent $(m)$ increases from -2 to 0.5. Also, it is shown that an increase in the magnetic intensity stabilizes the fluid flow in the channel. The results revealed among others, the thermal criticality conditions and with right combination of thermophysical parameters controlling the system, the thermal runaway can be prevented.

\section{References}

[1] Adiutori E. P. (1992): The Derivation and Application of Criteria for the Thermal Stability of Heat Transfer Media, HTD - Vol. 204, 89 - 95.

[2] Okoya S. S. (2006): Thermal stability for a Reactive Viscous Flow in a Slab, Mechanics research Communication 33, 728 -733 .
[3] Herron I. H. and Soliman F. S. (2006): The Stability of Couette Flow in a Torodial Magnetic Field, Applied Mathematics Letters 19, 1113 - 1117.

[4] Makinde O. D. and Maserumule M. (2010): Inherent Irreversibility and Thermal Stability for Steady Flow of a Variable Viscosity Liquid Film in a Cylindrical Pipe with Convective Cooling at the Surface, International Journal of Numerical Methods for Heat and Fluid Flow, Vol. 20 No. 1, 5 $-16$.

[5] Adesanya S.O. (2013), Thermal Stability Analysis of a Reactive Hydromagnetic Third - Grade Fluid through a Channel with Convective Cooling, Journal of the Nigerian Mathematical Society Vol. 32, 61 - 72.

[6] Makinde O. D. and Anwar Beg O. (2010): On inherent Irreversibility in a Reactive Hydromagnetic Channel Flow, Journal of Thermal Science Vol. 19 No. 1, 72 - 79.

[7] Frank Kamenettsk D. A. (1969), Diffusion and heat transfer in chemical kinetics, plenum press, New York.

[8] Wazwaz A. M. and El-Sayed ((2001)): A new modification of the Adomian decomposition Method for linear and nonlinear operators, Journal of Applied Maths Computation, 122, $393-405$.

[9] Hassan A. R. and Fenuga O.J. (2011), Flow of a Maxwell Fluid through a Porous Medium Induced by a constantly accelerating Plate, Journal of the Nigerian association of Mathematical Physics, Vol. 19, $249-254$

[10] Adesanya S. O. and Gbadeyan J. A. (2011): Adomian Decomposition approach to Steady visco - elastic Fluid flow with slip through a planer channel, International Journal of Nonlinear Science, Vol. 11, No. 1, 86 - 94.

[11] Gbadeyan J.A. and Hassan A.R. (2012): Multiplicity of Solutions for a reactive variable viscous Couette flow under Arrhenius Kinetics, Mathematical Theory and Modelling, Vol. 2, No.9, $39-49$

[12] Hassan A. R and Gbadeyan J.A. (2013): The Effect of Heat Absorption on a Variable Viscosity Reactive Couette Flow under Arrhenius Kinetics, Theoretical Mathematics \& Applications. Vol. 3, No.1 $145-159$

[13] Adesanya S.O. and Ayeni R. O. (2011), Existence and Uniqueness Result for couple stress Bio - Fluid Flow Model via Adomian Decomposition Method, International Journal of Nonlinear Science, Vol. 12, No. 1, 16-24. 